\title{
Miocardiopatía dilatada por miocarditis aguda: ¿Bordetella pertussis como agente causal?
}

\section{Dilated miocardiopathy after acute miocarditis: Bordetella pertussis as an etiological agent?}

Marcos Clavero-Adell, Daniel Palanca-Arias, Ariadna Ayerza-Casas, Marta López-Ramón, Lorenzo JiménezMontañés

Resumen

ANTECEDENTES: La miocardiopatía dilatada es la causa principal de trasplante cardiaco en niños.

CASO CLínICO: Paciente de 4 meses deedad que nació a las 29 semanas de gestación con miocardiopatía dilatada secundaria a infección por Bordetella pertussis. Al establecer el diagnóstico se encontraron factores pronóstico desfavorables (edad del paciente, cifra inicial elevada de proBNP y fracción de eyección del ventrículo izquierdo menor de $30 \%)$; sin embargo, después de seis meses de tratamiento inmunodulador, con ciclosporina y prednisona, tuvo evolución clínica adecuada.

CONCLUSIONES: Si bien la miocardiopatía dilatada es una alteración que pone en riesgo la vida de los pacientes, la evolución y recuperación del paciente de este caso fue satisfactoria durante el primer año de seguimiento.

PALABRAS CLAVE: Miocardiopatía dilatada; miocarditis aguda; Bordetella pertussis.

Abstract

BACKGROUND: Dilated cardiomyopathy is the main cause of pediatric cardiac transplantation.

CLINICAL CASE: We present a four months old child (preterm birth at 29-week gestational age) affected from dilated cardiomyopathy secondary to Bordetella pertussis infection. At diagnosis there are unfavorable prognostic factors (patient age, high initial proBNP figure and left ventricular ejection fraction less than 30\%). However, after 6 months of immunomodulatory treatment with ciclosporine and prednisone he presents an adequate clinical evolution.

CONCLUSIONS: Although dilated cardiomyopathy is an alteration that puts patients' lives at risk, it is worth noting the satisfactory clinical and analytical evolution and recovery of our case during the first year.

KEYWORDS: Dilated cardiomyopathy; Acute myocarditis; Bordetella pertussis
Hospital Universitario Miguel Servet, Zaragoza, España.

Recibido: 19 de junio 2019

Aceptado: 9 de enero 2020

Correspondencia

Marcos Clavero Adell

m.claveroadell@gmail.com

Este artículo debe citarse como

Clavero-Adell M, Palanca-Arias D, Ayerza-Casas A, López-Ramón $M$, Jiménez-Montañés L. Miocardiopatía dilatada por miocarditis aguda: ¿Bordetella pertussis como agente causal? Acta Pediatr Mex. 2020;41(1):21-5. 


\section{ANTECEDENTES}

La miocarditis aguda es una enfermedad inflamatoria provocada por diversos factores: desde formas subclínicas hasta miocardiopatía dilatada secundaria, choque cardiogénico o muerte súbita. ${ }^{1}$ Si bien el diagnóstico de confirmación se establece con base en el reporte histológico deben integrarse los criterios clínicos, analíticos, electrocardiográficos y de imagen. ${ }^{2}$ Se han descrito múltiples causas de miocarditis en pacientes pediátricos; no obstante, la infección representa el principal motivo de incidencia en estos casos. ${ }^{3}$

\section{CASO CLÍNICO}

Lactante masculino, de 4 meses de edad, con signos y síntomas de tos, dificultad respiratoria y rechazo a la alimentación de dos días de evolución. Antecedentes de prematuridad (29 semanas de edad gestacional), con peso al nacimiento de $1160 \mathrm{~g}$, displasia broncopulmonar leve e infección respiratoria (bronquiolitis leve) al tercer mes de vida. Fue valorado en Urgencias de Pediatría, donde se apreciaron: taquipnea, tiraje e hipoventilación del hemitórax izquierdo. La radiografía de tórax (Figura 1A) reportó atelectasia de pulmón izquierdo. Se completó el estudio con tomografía de tórax, que evidenció cardiomegalia masiva a expensas de cavidades izquierdas y atelectasia subtotal del pulmón izquierdo, probablemente secundaria a compresión cardiaca. Figura 1B

La ecocardiografía mostró el ventrículo izquierdo muy dilatado (Figura 1C) y con alteración en la contractilidad: diámetro telediastólico del ventrículo izquierdo de $41 \mathrm{~mm}$ (Z-score +7.14 DE) y fracción de eyección del ventrículo izquierdo por el método de Simpson de 10\%. El análisis sanguíneo reportó: pro-BNP de 12,500pg/mL, con troponina ultrasensible negativa.

Con el diagnóstico de miocardiopatía dilatada, con insuficiencia cardiaca congestiva, ingresó a la Unidad de Cuidados Intensivos Pediátricos. Se inició tratamiento para insuficiencia cardiaca

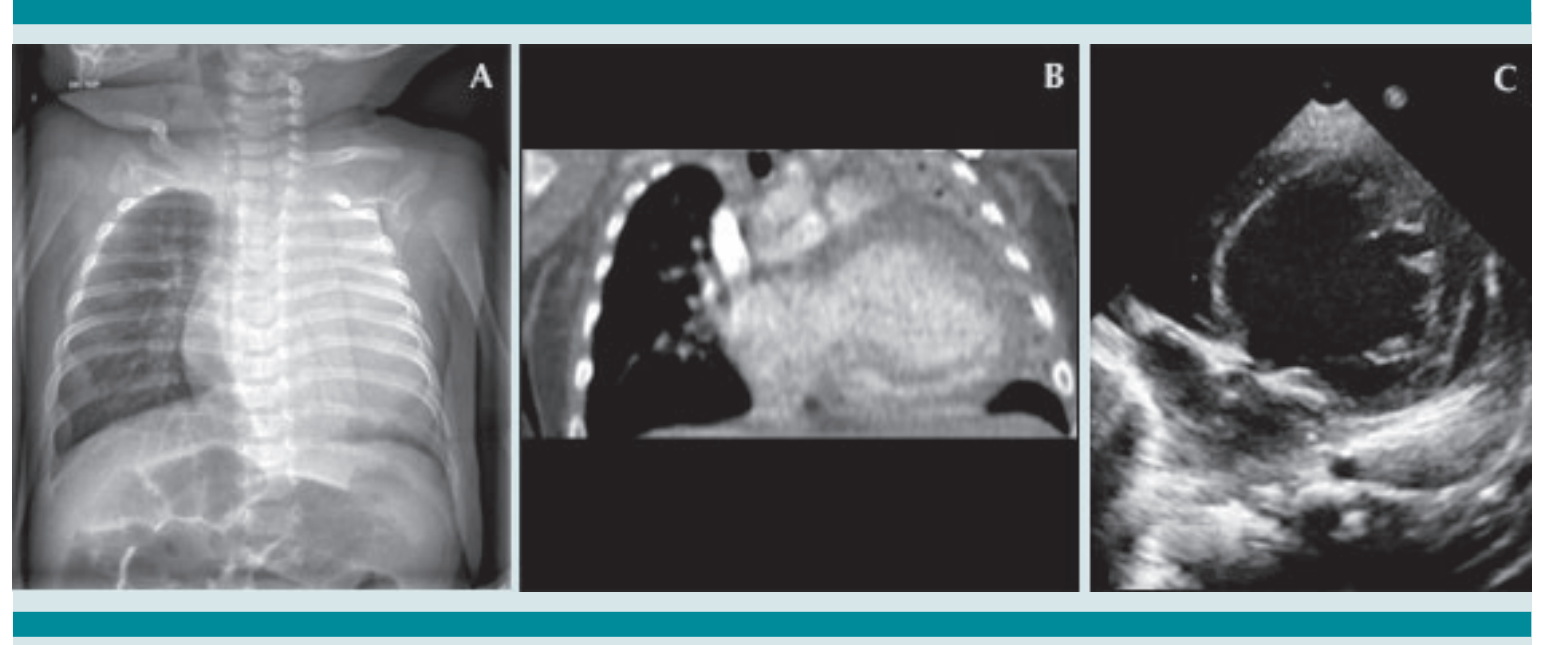

Figura 1. A) Radiografía de tórax que evidencia opacidad en el hemitórax izquierdo compatible con atelectasia pulmonar. B) Tomografía del tórax que muestra cardiomegalia a expensas de la dilatación de las cavidades izquierdas. C) Ecocardiografía transtorácica que reporta una dilatación grado VI. 
con captopril, furosemida, espironolactona y milrinona, que permitió que el soporte inotrópico se suspendiera a los seis días del ingreso. Se trató de forma empírica el posible cuadro con inmunoglobulinas inespecíficas humanas intravenosas, biotina, carnitina y tiamina. Después de la estabilización inicial se prescribió carvedilol. El panel genético de miocardiopatías familiares y los estudios hormonal (eje hipotálamo-hipofisario, suprarrenal, tiroideo) y metabólico (errores congénitos del metabolismo) resultaron normales, por lo que se suspendió el suplemento vitamínico. En un amplio estudio de enfermedades infecciosas se aisló Bordetella pertussis por PCR en el aspirado nasofaríngeo. Se trató con azitromicina.

Se desestimó efectuar resonancia magnética cardiaca y biopsia endomiocárdica debido al elevado riesgo anestésico. A los 15 días se inició el tratamiento inmunosupresor con prednisona a dosis decrecientes (inicial de $2 \mathrm{mg} / \mathrm{kg} /$ día y de mantenimiento $0.1 \mathrm{mg} / \mathrm{kg} /$ día) y ciclosporina $(1 \mathrm{mg} / \mathrm{kg} / \mathrm{d}$ ía) durante seis meses. Fue dado de alta a su domicilio a los 21 días del ingreso. Los estudios de control sucesivos reportaron mejoría clínica, ecocardiográfica y analítica progresiva. (Figura 2) Siete meses después, en tratamiento con carvedilol, desaparecieron los síntomas y las cifras de Pro-BNP se encontraron en los límites de referencia; los estudios de imagen no reportaron alteraciones y se observó reducción del diámetro telediastólico del ventrículo izquierdo de 41 a $22 \mathrm{~mm}$.

\section{DISCUSIÓN}

La miocardiopatía dilatada es la dilatación y disfunción del ventrículo izquierdo. Representa la forma principal de miocardiopatía y la causa más común de trasplante cardiaco en niños. ${ }^{4}$ La miocarditis infecciosa es una de los principales fuentes de miocardiopatía dilatada en la población pediátrica. Las infecciones virales, ya sea por lesión directa o reacción inmunitaria, son la principal causa de esta alteración; ${ }^{5}$ sin embargo, se han descritos casos por bacterias, hongos, protozoarios o parásitos. ${ }^{6}$

Después del exhaustivo y extenso estudio microbiológico, hormonal, metabólico, tóxico, enfermedades sistémicas, etc., se identificó Bordetella pertussis en este paciente, que resulta un caso excepcional, porque no se encuentran descritos reportes similares en la bibliografía. Aunque la evolución fue favorable después del
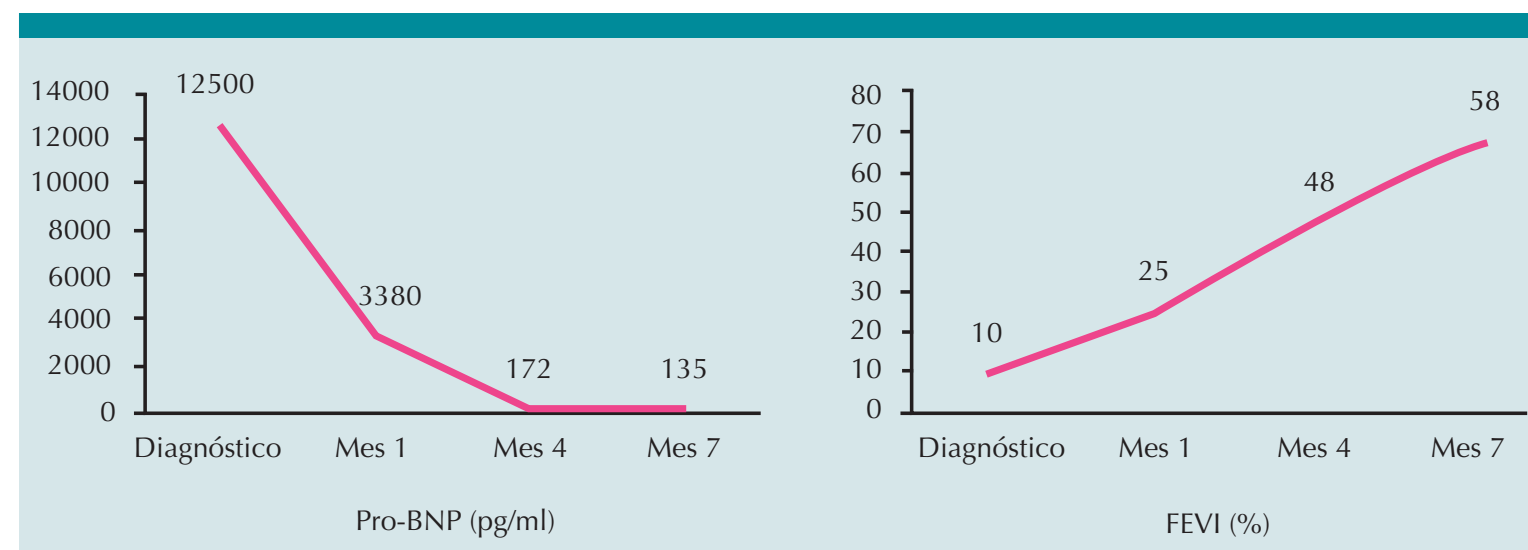

Figura 2. Evolución del pro-BNP y fracción de eyección del ventrículo izquierdo al diagnóstico. 
tratamiento inmunomodulador, no puede descartarse como agente causante de la enfermedad. Entre los antecedentes clínicos debe considerarse el cuadro referido de infección respiratoria, un mes antes catalogado como bronquiolitis leve, el posible ambiente epidémico familiar, la prematuridad (29 semanas de gestación) y que en México la vacunación en la embarazada inicia en la semana 20; sin embargo, la madre no había recibido la vacuna contra Bordetella pertussis, recomendada durante esta etapa de forma estandarizada, entre las semanas 28 y 32 del embarazo. ${ }^{7}$

Aunque el diagnóstico definitivo de miocarditis aguda se confirma mediante biopsia endomiocárdica, es una prueba invasiva no exenta de complicaciones graves, que puede ofrecer falsos negativos, por lo que debe individualizarse cada caso e indicarse solo en pacientes críticos o con reacción deficiente al tratamiento inicial. ${ }^{8}$ Por ello, el diagnóstico debe establecerse con criterios clínicos y estudios de imagen, apoyados por pruebas complementarias que ayuden a identificar la causa de la enfermedad. La resonancia magnética es el estudio de elección para el diagnóstico de miocarditis aguda; ${ }^{9}$ sin embargo, se desestimó inicialmente, por la edad del paciente, quien requería sedación y supone un procedimiento de alto riesgo. Pudo efectuarse a los 14 meses de edad, con desenlaces congruentes con el estudio ecocardiográfico.

El tratamiento inicial en pacientes con miocarditis aguda incluye diuréticos, inotropos, reductores de la poscarga y antiarrítmicos, además de la búsqueda del agente causal u origen real de la enfermedad. ${ }^{3}$ En pacientes con miocarditis negativa por virus, después del periodo inicial inflamatorio agudo, debe mantenerse el tratamiento de la insuficiencia cardiaca (si fuera necesario) y se recomienda el tratamiento con inmunosupresores, fundamentalmente basados en prednisona y ciclosporina, ${ }^{10}$ o prednisona y azatioprina, ${ }^{11}$ pues han demostrado aumentar la supervivencia y la fracción de eyección del ventrículo izquierdo a seis meses.

Algunos estudios indican, durante la hospitalización inicial, parámetros pronóstico que podrían ayudar a identificar a los pacientes de alto riesgo, principalmente: mediciones ecocardiográficas (fracción de acortamiento, FEVI menor de $30 \%$, presiones de llenado auricular), hemodinámicas y séricas (proBNP > 2000 pg/ $\mathrm{mL}$ con buena correlación y peor FEVI, colesterol sérico, ácido úrico y saturación venosa mixta), incluso factores como: edad menor de 2 años al ingreso o curso prolongado de la enfermedad. ${ }^{5}$ Además del tratamiento médico, una proporción de pacientes requerirá trasplante cardíaco en los primeros 12 meses del ingreso hospitalario. ${ }^{12}$

La vacunación contra Bordetella pertussis durante el embarazo es efectiva y segura. ${ }^{13}$ Puesto que la infección por Bordetella pertusis va en aumento y los recién nacidos prematuros y con problemas respiratorios tienen mayor riesgo de complicaciones graves a causa de la tos ferina, la inmunización a todas las embarazadas debe continuar y recibir todo el apoyo posible. ${ }^{14}$

\section{CONCLUSIONES}

Si bien la miocardiopatía dilatada es una alteración que pone en riesgo la vida de los pacientes, la evolución y recuperación clínica y analítica satisfactoria de nuestro caso, a lo largo del primer año, pese a los datos iniciales de mal pronóstico (edad del paciente, cifra inicial elevada de proBNP y fracción de eyección del ventrículo izquierdo menor de $30 \%$ ). 
Clavero- Adell M, et al. Miocardiopatía dilatada y Bordetella pertussis

\section{REFERENCIAS}

1. Moonen M, et al. Update on myocarditis. Rev Med Liege 2018; 73(5-6):269-276. https://www.rmlg.ulg.ac.be/ show.php

2. Elamm C, et al. Republished: Pathogenesis and diagnosis of myocarditis. Postgrad Med J 2012; 88(1043):539544. https://www.ncbi.nlm.nih.gov/pmc/articles/ PMC5813801/

3. Canter $\mathrm{CE}$, et al. Diagnosis and treatment of myocarditis in children in the current era. Circulation 2014;7;129(1):11528. https://www.ahajournals.org/doi/full/10.1161/CIRCULATIONAHA.113.001372

4. Towbin JA, et al. Incidence, causes, and outcomes of dilated cardiomyopathy in children. JAMA 2006;18;296(15):186776. DOI: 10.1001/jama.296.15.1867

5. Rodriguez-Gonzalez M, et al. Clinical presentation and early predictors for poor outcomes in pediatric myocarditis: a retrospective study. World J Clin Cases 2019;6;7(5):548561. DOI: 10.12998/wjcc.v7.i5.548

6. Caforio ALP, et al. Current state of knowledge on aetiology, diagnosis, management, and therapy of myocarditis: A position statement of the European Society of Cardiology Working Group on Myocardial and Pericardial Diseases. Eur Heart J 2013;34(33):2636-48, 2648a-2648d. doi: 10.1093/ eurheartj/eht210.

7. Campbell $\mathrm{H}$, et al. Review of vaccination in pregnancy to prevent pertussis in early infancy. J Med Microbiol 2018;67(10):1426-1456. doi: 10.1099/jmm.0.000829.
8. Das BB. Role of endomyocardial biopsy for children presenting with acute systolic heart failure. Pediatr Cardiol 2014;35(2):191-6. doi: 10.1007/s00246-013-0807-0.

9. Sachdeva $S$, et al. Analysis of clinical parameters and cardiac magnetic resonance imaging as predictors of outcome in pediatric myocarditis. Am J Cardiol 201515;115(4):499-504. doi: 10.1016/j.amjcard.2014.11.029.

10. Gagliardi MG, et al. Long term follow up of children with myocarditis treated by immunosuppression and of children with dilated cardiomyopathy. Heart 2004;90(10):1167-71. DOI: 10.1136/hrt.2003.026641

11. Frustaci $A$, et al. Randomized study on the efficacy of immunosuppressive therapy in patients with virusnegative inflammatory cardiomyopathy: The TIMIC study. Eur Heart J 2009;30(16):1995-2002. doi: 10.1093/ eurheartj/ehp249

12. Hollander SA, et al. Outcomes of children following a first hospitalization for dilated cardiomyopathy. Circ Heart Fail 2012;5(4):437-43. doi: 10.1161/CIRCHEARTFAILURE.111.964510.

13. Furuta $M$, et al. Efficacy and safety of pertussis vaccination for pregnant women - a systematic review of randomised controlled trials and observational studies. BMC Pregnancy Childbirth 2017;17(1):390. doi: 10.1186/ s12884-017-1559-2

14. Byrne $L$, et al. Hospitalisation of preterm infants with pertussis in the context of a maternal vaccination programme in England. Arch Dis Child 2018;103(3):224-229. doi: 10.1136/archdischild-2016-311802. 